Helgoländer wiss. Meeresunters. 25, 14-25 (1973)

\title{
Striaria attenuata (Phaeophyta, Dictyosiphonales), neu bei Helgoland: Entwicklung und Aufbau
}

\author{
P. Kornmann \& P.-H. Sahling \\ Biologische Anstalt Helgoland (Meeresstation); \\ Helgoland, Bundesrepublik Deutscbland
}

\begin{abstract}
Striaria attenuata (Phaeophyta, Dictyosiphonales), newly found at Helgoland; development and structure. S. attenuata was first recorded at Helgoland (North Sea) in 1971. Contrary to the rather complicated life cycle of French material, only asexual generations followed one another in cultures. Zoospores of unilocular sporangia develop into a creeping protonema, from which upright Striaria-shoots arise at an age of 3 weeks. Apical growth of protonema filaments changes into an intercalary growth pattern in Striaria-shoots. Howeyer, it is remarkable and cannot be explained, why only a low rate of about 1 in 70 protonemata develop in this way. As the shoots grow very quickly, details of development and structure could be elucidated; there is no meristematic zone, but the whole axis lengthens intercalary until fertilization.
\end{abstract}

\section{EINLEITUNG}

Ausgangsmaterial für diese Studie waren zwei erstmalig bei Helgoland am 1. Juli 1971 gefundene Striaria-Pflanzen. Sie wuchsen auf der Betonwand eines künstlichen Gezeitenbeckens, waren etwa $3,5 \mathrm{~cm}$ hoch und reich fertil, so daß Kulturen angelegt werden konnten.

Über die Entwicklung von Striaria attenuata haben KyLIN (1934) und CARAM (1965) berichtet. Kyin untersudate Material von der schwedischen Westküste; aus den Schwärmern der unilokulären Sporangien erhielt er dichte Fadenbüschel. „Nach vier Wochen waren in den Kulturen kleine Pflanzen von Striaria attenuata vorhanden. Diese hatten sich in rein vegetativer Weise aus kleinen Seitenzweigen entwickelt" (KYLIN 1934, p. 15). Ohne fertil geworden zu sein, starben die 7 Wochen alten Kulturen $a b$.

Ganz andersartig und dazu noch völlig uneinheitlich ist der Lebenszyklus der von CARAM untersuchten Striaria von den Küsten der Bretagne. Ein Teil der Schwärmer aus den unilokulären Sporangien verhielt sich wie Gameten; aus der Zygote entstand ein Protonema als Träger von Striaria-Sprossen. Diesen direkten monogenetischen Diplontenzyklus zeigten 30 bis $40 \%$ der in einer Kultur aufwachsenden Keimstadien. Die nicht kopulierenden Schwärmer entwickelten sich zu Prothallien mit plurilokulären Sporangien. Auch deren Schwärmer konnten verschmelzen oder sich unmittelbar ent- 

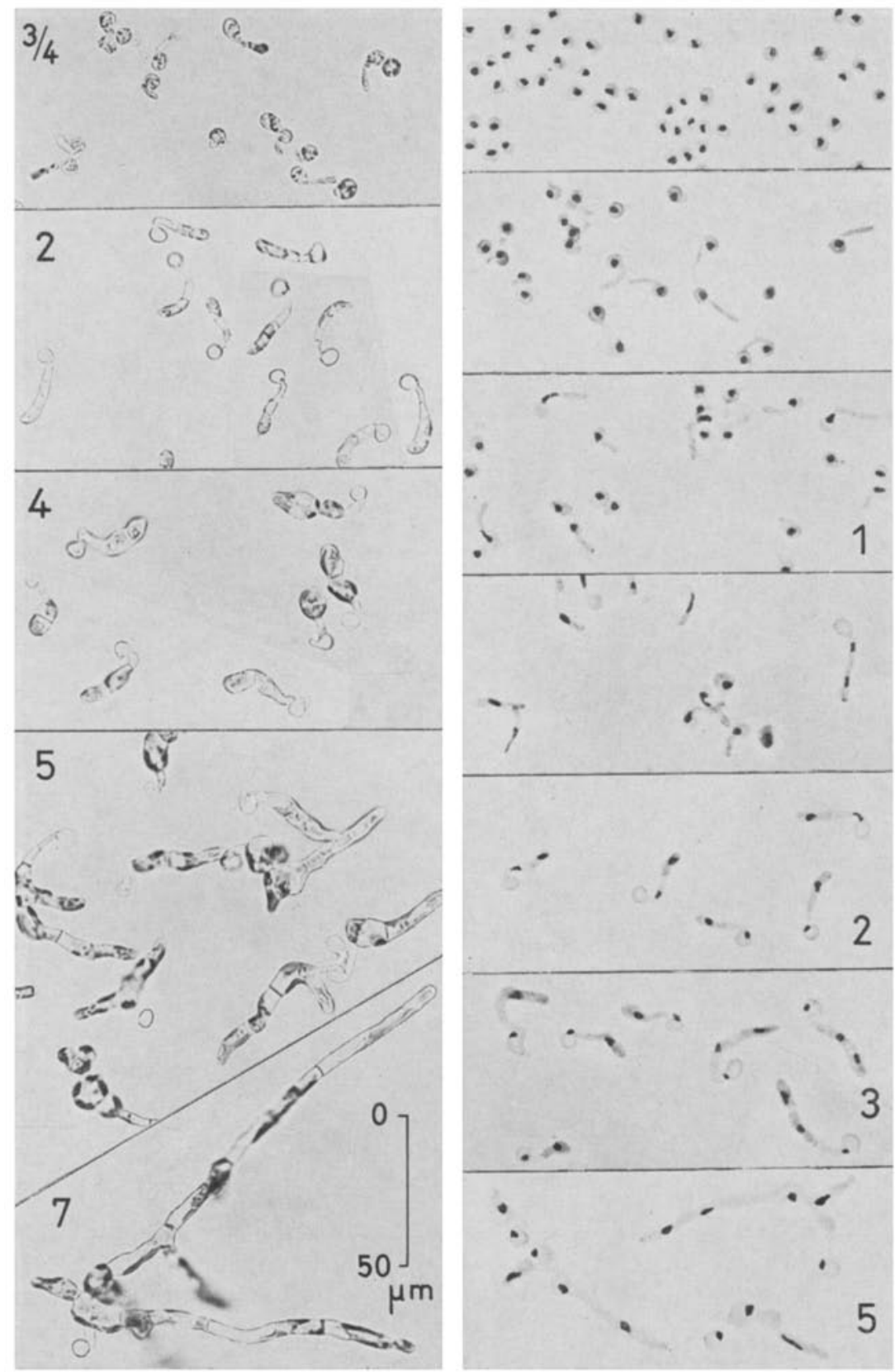

Abb. 1: Striaria attenuata. Keimung der Zoosporen und Entwicklung der Protonemata, lebend (links) und nach Färbung mit Karminessigsäure (rechts). Die Zahlen geben das Alter in Tagen an 

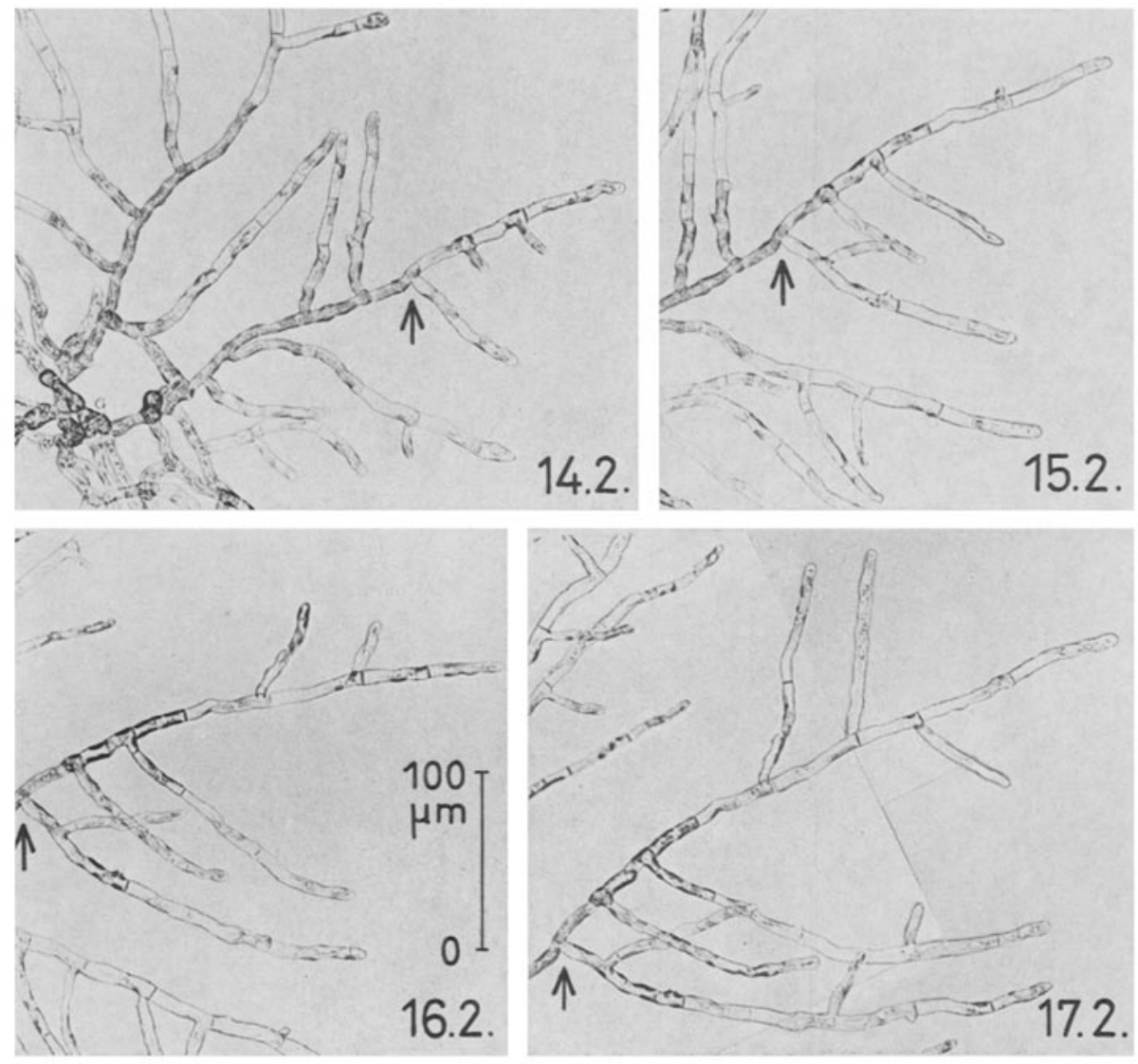

Abb. 2: Striaria attenuata. Spitzenwachstum des Protonemas. Aufnahmen in Tagesabständen; der Pfeil bezeichnet jeweils die gleiche Stelle

widkeln. Aus den Zygoten entstand eine Striaria-Generation: der Lebenszyklus ist in diesem Falle diplohaplontisch. Die unverschmolzenen Schwärmer ergaben Plethysmothallien mit plurilokulären Sporangien. Ihre Schwärmer kopulierten nicht; es reihten sich mehrere derartige Generationen aneinander. Ob sie gelegentlich wieder $\mathrm{zu}$ Geschlechtspflanzen werden können, ist nicht bekannt. Die Striaria-Pflanze kann also ebenso wie das Prothallium zugleich Gametophyt und Sporophyt sein.

Bei diesen regionalen Verschiedenheiten erschien es wünschenswert, das Verhalten der Helgoländer Striaria zu prüfen. Um das Ergebnis vorwegzunehmen: ihr Lebenszyklus entspricht dem des von KYIIN untersuchten Materials. Die leichte Kultivier-" barkeit - es wurden in kurzer Zeit mehrere Generationen stattlicher fertiler Pflanzen aufgezogen - ermöglichte es, Einzelheiten ihrer Entstehungsgeschichte genauer zu be-obachten. Auf dieser Grundlage läßt sich der morphologische Aufbau von Striaria leicht verstehen. 

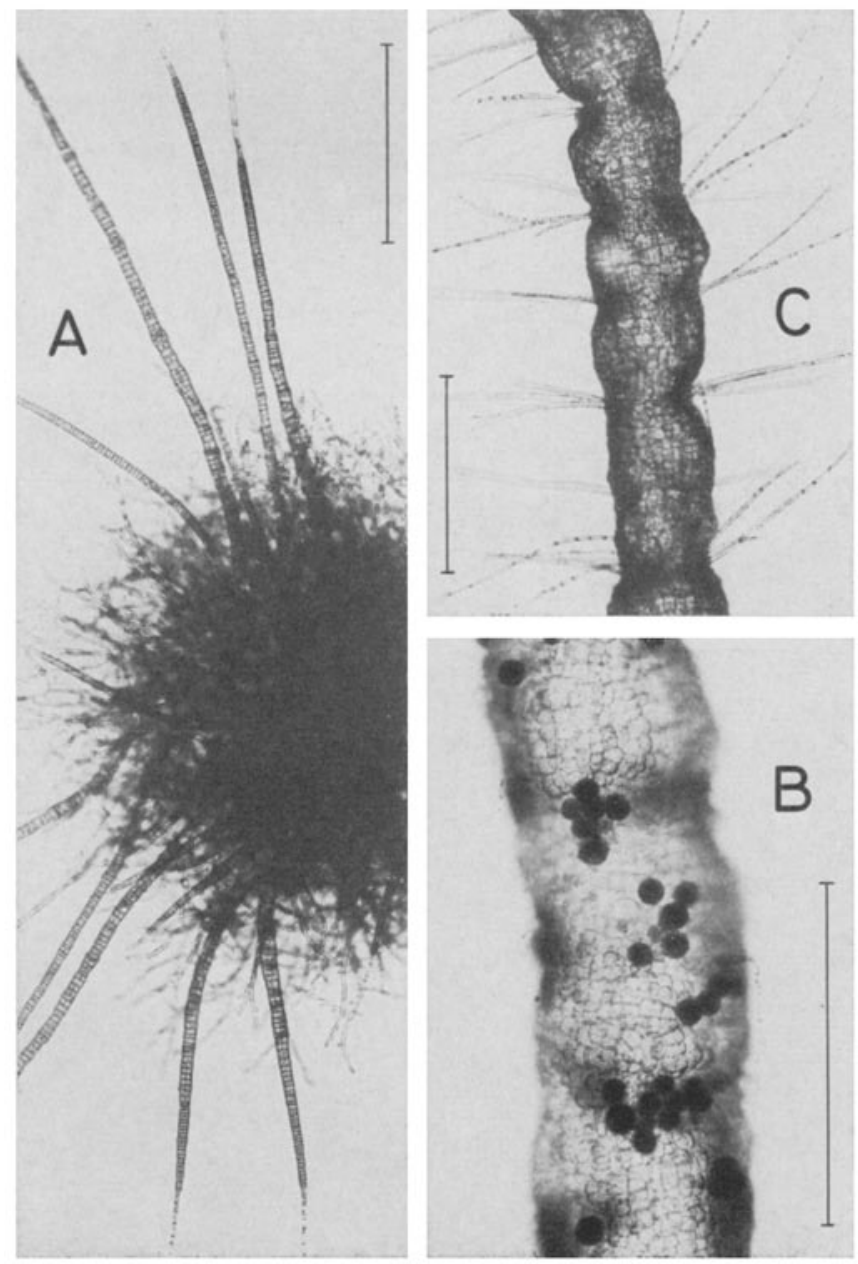

Abb. 3: Striaria attenuata. A 22 Tage altes Protonema mit aufrechten Thalli. $B$ Ausschnitt aus einem fertilen Thallus. $C$ Thallusstück mit Haarwirtein und Einschnürungen. Die eingetragenen Strecken entsprechen jeweils $0,5 \mathrm{~mm}$

\section{KULTURVERSUCHE}

\section{Der Lebenszyklus}

Die Schwärmer der unilokulären Sporangien keimen alle in gleicher Weise. Kopulationen wurden niemals beobachtet; die kurz nach dem Festsetzen gefärbten Zoosporen waren alle einkernig (Abb. 1). Bei den meisten ist der Inhalt schon am nächsten Tage in einen Keimschlauch übergetreten, und der in ihm liegende langgestreckte Kern teilt sich. Zwei Tage alte Stadien sind bereits zweikernig; einer der Kerne ist in die fast 
inhaltslose und durch die erste Querwand abgetrennte Embryospore zurückgewandert, er teilt sich nicht mehr. Bis zum 5. Tage schwellen die Zellen des inzwischen zwei- bis dreizellig gewordenen Keimlings stark an; an solchen und älteren Stadien ist der Keimschlauch mit der Embryospore deutlich erkennbar abgesetzt. Danach beginnt ein sehr

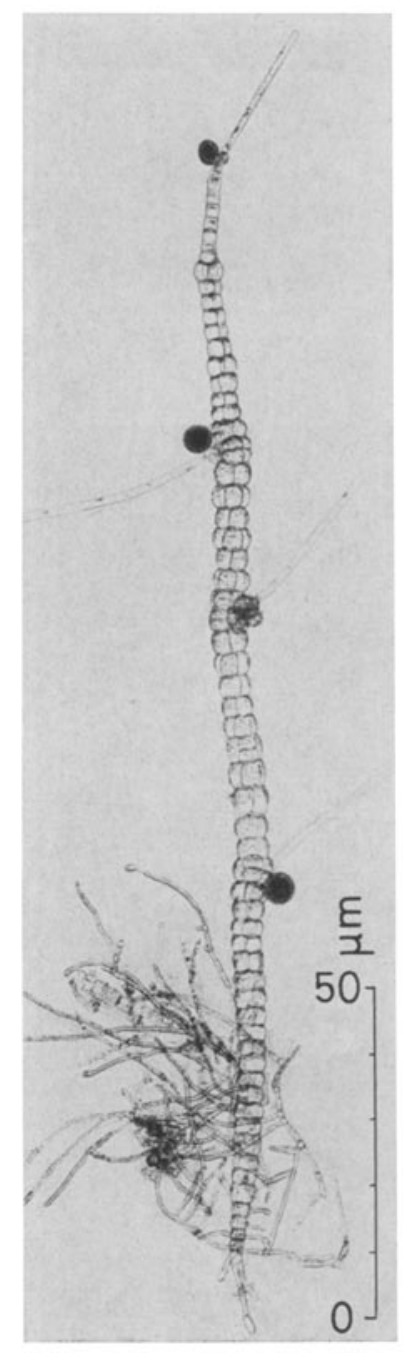

Abb. 4: Striaria attenuata. Kümmerform, aus einer 7 Wochen alten Kultur inmitten vieler Protonemata

lebhaftes Wachstum, das zu einem einreihigen, reich verzweigten Protonema führt (Abb. 2). Nur die Apikalzellen dieser Fäden sind streckungsfähig, sie wachsen mit gleichmäßiger Geschwindigkeit und gliedern Fadenzellen ab. Verzweigungen entspringen stets unter der oberen Querwand, im allgemeinen in akropetaler Folge, so daß die 
Protonema-Fäden monopodial mit deutlicher Führung der Hauptachse aufgebaut sind. Das in Abbildung 2 in Tagesabständen photographierte Fadenende ist ein kleiner Ausschnitt aus einem Protonema, das bei Beginn der Beobachtung 12 Tage alt war und einen Durchmesser von etwa $0,6 \mathrm{~mm}$ hatte. Während der dreitägigen Beobachtung unter einem Deckglas war keine Beeinträchtigung des Wachstums zu erkennen.

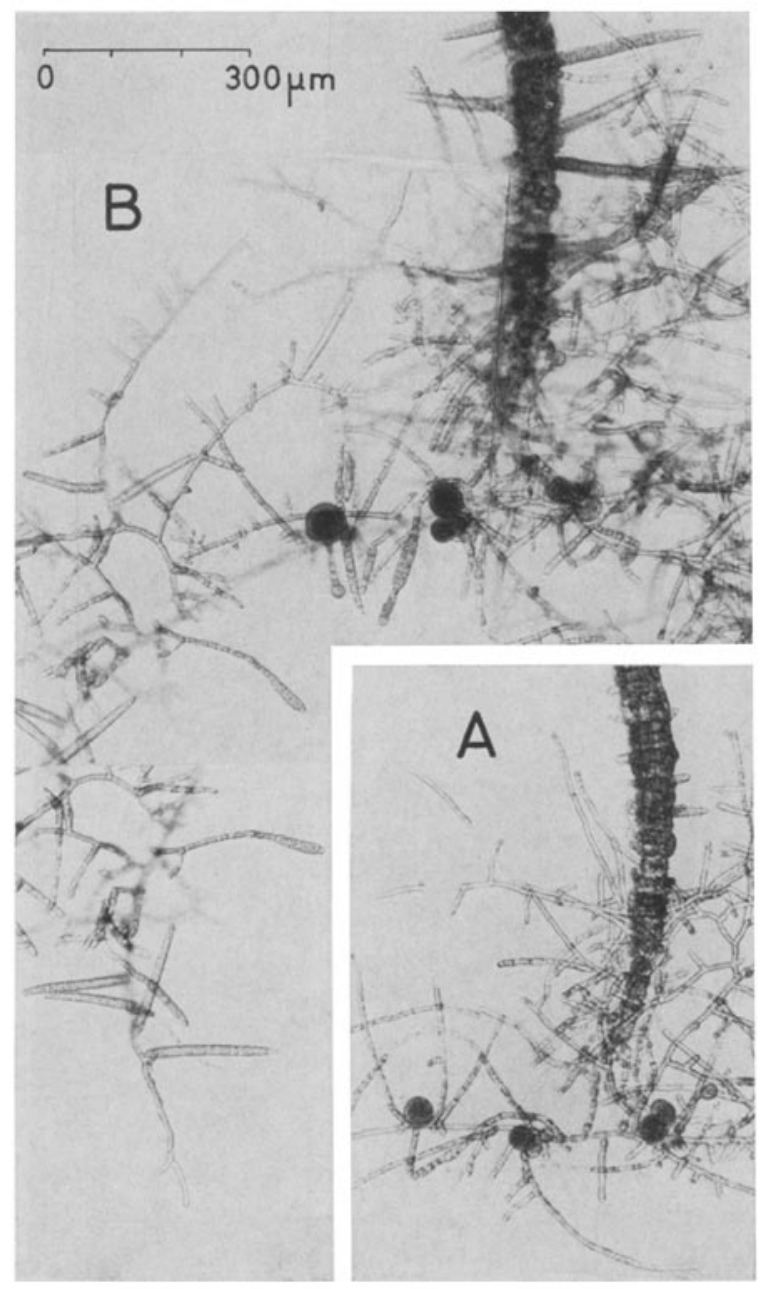

Abb. 5: Striaria attenuata. A Protonema mit unilokulären Sporangien und aufrechtem Thallus. $B$ Drei Tage späzer mit zahlreichen jungen Striaria-Sprossen

Nach 3 bis 4 Wochen sind die Protonemata zu flachen bis halbkugeligen, reidiver$z$ weigten Polstern von rund $3 / 4 \mathrm{~mm}$ Durchmesser herangewachsen; einzelne tragen dann schon gut erkennbare aufrechte Striaria-Thalli (Abb. 3 A). Die ersten Sprosse konnten an 17 Tage alten Pflänzchen gefunden werden. 
Hier muß auf einen wesentlichen Befund mit allem Nachdruck hingewiesen werden: die Striaria-Sprosse entstehen nur auf einem ganz geringen Anteil der Protonemata. Im Durchschnitt trägt jedes 70 . Protonema aufrechte Thalli, häufig nur einen, gelegentlich aber bis zu vierzig. Wie sich KyLINs Kulturen in dieser Hinsicht verhielten, ist leider nicht bekannt. An mehr als 5 Wochen alten Protonemata wurden keine Striaria-Sprosse mehr gebildet, auch nicht nach Erneuerung der Kulturen oder auf Regeneraten fein zerteilter Polster. Aus welchen Gründen immer nur einige wenige der in einer Kulturschale unter völlig gleichen Bedingungen aufgewachsenen Protonemata aufrechte Sprosse bilden, ist unbekannt.

Stattliche Striaria-Pflanzen erhält man nur, wenn Protonemata mit aufrechten Sprossen möglichst frühzeitig - etwa in dem Stadium der Abbildung 3 A - isoliert und in frischer Nährlösung weiterkultiviert werden. Sonst bleiben die Sprosse dünn und unverzweigt, die ursprünglich monosiphone Achse wird nur durch wenige Längswände aufgeteilt; die Pflänzchen werden höchstens $1,5 \mathrm{~cm}$ lang und spärlich fertil (Abb. 4). Unter optimalen Bedingungen dagegen wachsen innerhalb von 4 Wochen bis zu $10 \mathrm{~cm}$ große, reich verzweigte und fertile Thalli heran (Abb. $3 \mathrm{~B}$ ). In ruhigstehenden Kulturen sind die in charakteristischer Weise eingeschnürten Thalli von dichten Haarwirteln besetzt (Abb. 3 C). Die Fertilisierung schreitet von der Basis zur Spitze fort; selbst Seitenzweige zweiter Ordnung tragen schließlich unilokuläre Sporangien. Im allgemeinen werden nur die aufredten Thalli fertil; nur in einem Falle wurden unilokuläre Sporangien auch auf einem Protonema gefunden, das bereits einen Striaria-Sproß trug (Abb. 5 A). Drei Tage später erschienen auf dem sehr locker wachsenden Protonema junge Striaria-Sprosse in großer Zahl (Abb. $5 \mathrm{~B}$ ).

Kulturen mit reifen Pflanzen werden sehr bald durch die Masse der sich entwikkelnden Protonemata überständig, die den Schalenboden, die Oberfläche der Flüssigkeit und die Pflanzen selbst besiedeln. Es ist daher zweckmäßig, die Kulturen aus den Schwärmern der zuerst gereiften Sporangien zu erneuern. Auf diese Weise konnten fünf Generationen gleichartiger Pflanzen innerhalb 7 Monaten im Laboratorium aneinandergereiht werden. Als Nährlösung diente Erdschreiberlösung, die Kulturen wuchsen bei $15^{\circ} \mathrm{C}$ und einer täglich 14 stündigen Beleuchtung von rund $1000 \mathrm{Lux}$.

\section{Die Differenzierung des Striaria-Thallus}

Der aufrechte Striaria-Sproß entsteht aus einer „Knospe“, zu der sich ein Fadenende oder ein Zweig des Protonemas verdickt (Abb. 5B). In ihr geht das Spitzenwachstum der fädig-kriechenden Organisationsstufe zu dem interkalaren Wachstum des aufrechten Thallus ïber. Welche Faktoren diesen Entwicklungsvorgang auslösen, ist unbekannt. Umgekehrt können aus einem Striaria-Thallus wieder Rhizoide mit Spitzenwachstum hervorgehen, zum Beispiel an einer basalen Schnittfläche, wie auch gelegentlich an der Spitze eines wachsenden Thallus.

Der junge aufrechte Sproß ist wesentlich dicker als die Fäden des Protonemas. Er ist zunächst ein einreihiger Faden, dessen Zellen sich interkalar teilen, und der sich an der Spitze leicht verjüngt (Abb. 6). Hier erfolgt seine erste Differenzierung: schon frïhzeitig gliedert die inhaltsreiche Apikalzelle die Initiale für ein endständiges Haar ab, 


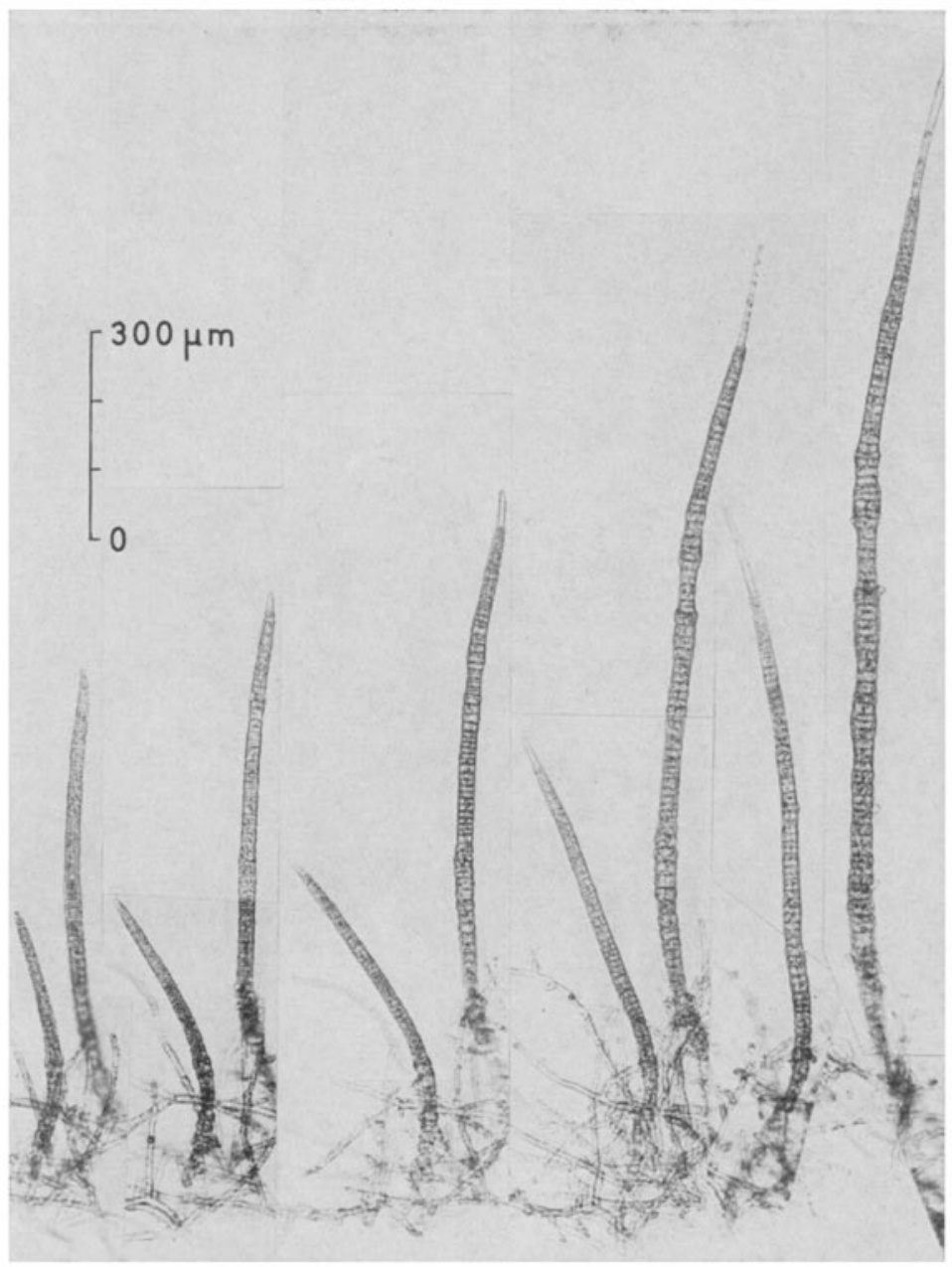

Abb. 6: Striaria attennata. Wachstum und Differenzierung junger Sprosse in Tagesabständen

wie dies bereits von REINKE (1878) beschrieben wurde. Als weitere morphologische Differenzierung entstehen Längswände in einzelnen, beliebig in dem Faden verteilten Zellen, zwischen denen zunächst noch monosiphone Abschnitte verbleiben. Durch das Wachstum der längsgeteilten Zellen erhält der Faden schon frühzeitig ein unregelmäßig knotiges, ganz individuelles Aussehen. Bald erscheinen an diesen Knoten Haare oder Seitenzweige, die als natürliche Marken die Sproßachse gliedern. An ihrer gegenseitigen Verschiebung läßt sich das Wachstum des Thallus klar erkennen.

Abbildung 7 zeigt die Wachstumsverteilung in der Hauptachse eines Thallus während einer 15tägigen Beobachtung. Die Achse streckt sich auf ihrer ganzen Länge interkalar; das Ausmaß der Streckung nimmt von der Basis zur Spitze zu. Mit der Fertilisierung, von der Basis zur Spitze fortschreitend, hört das Wachstum auf. Die beiden 


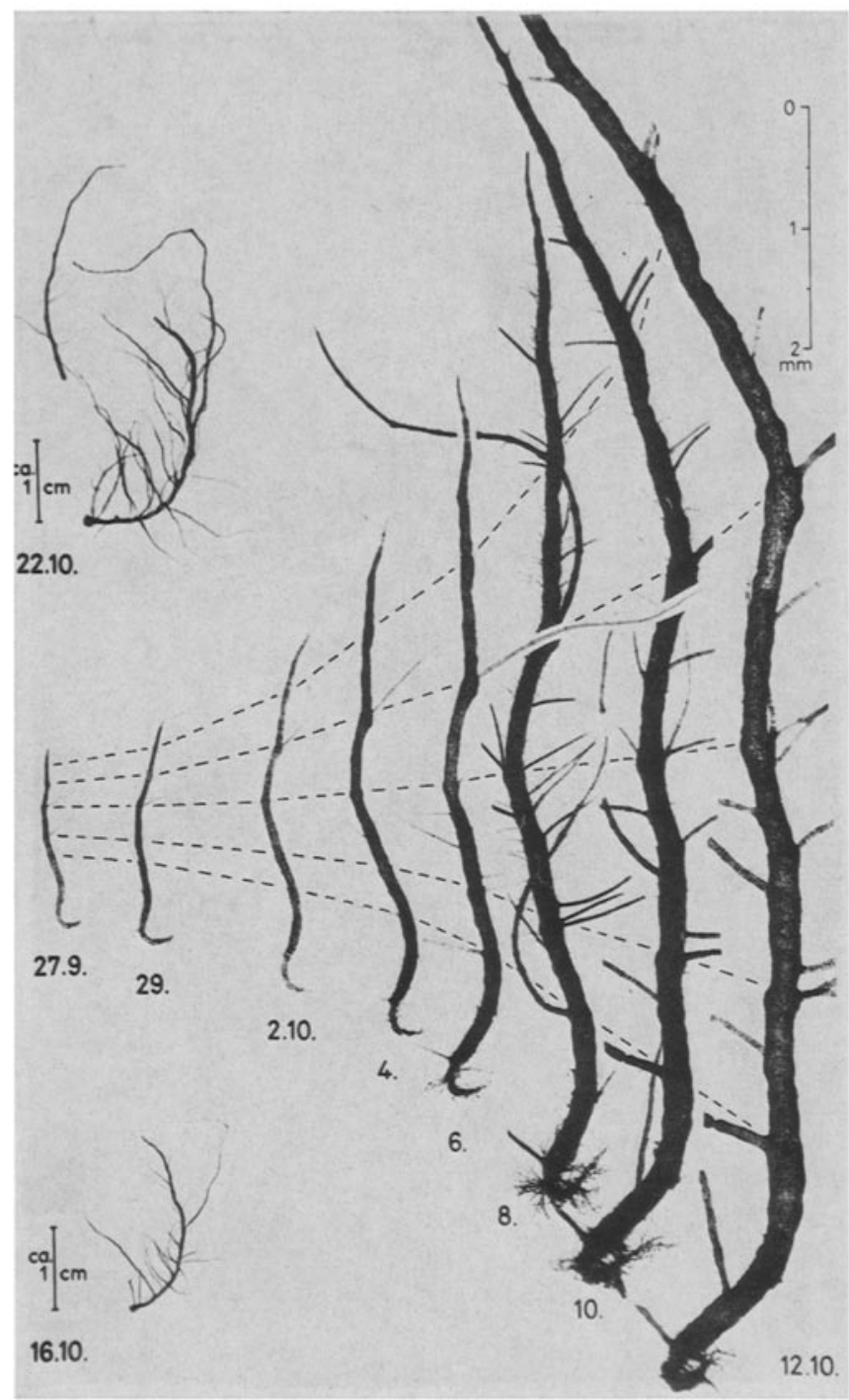

Abb. 7: Striaria attenuata. Wachstum einer Hauptachse während 15 Tagen; die ganze Pflanze 4 Tage (links unten) beziehungsweise 10 Tage (links oben) später dargestell

Habitusbilder links unten und oben zeigen die ganze Pflanze nach weiteren vier beziehungsweise zehn Tagen. Die Spitze der Hauptachse war schon nach der Aufnahme am 6. 10. verlorengegangen; der am 16. 10. nahe der Basis an einer starken Einschnürung abgebrochene Seitenzweig ist selbständig weitergewachsen.

Die Streckung eines Endabschnittes ist in einer 13tägigen Beobachtungsreihe dargestellt (Abb. 8). Alle Segmente des stets klar markierten Thallusendes strecken sich gleichmäßig; keine Zone zeichnet sich durch eine gesteigerte Teilungstätigkeit aus. Bis 


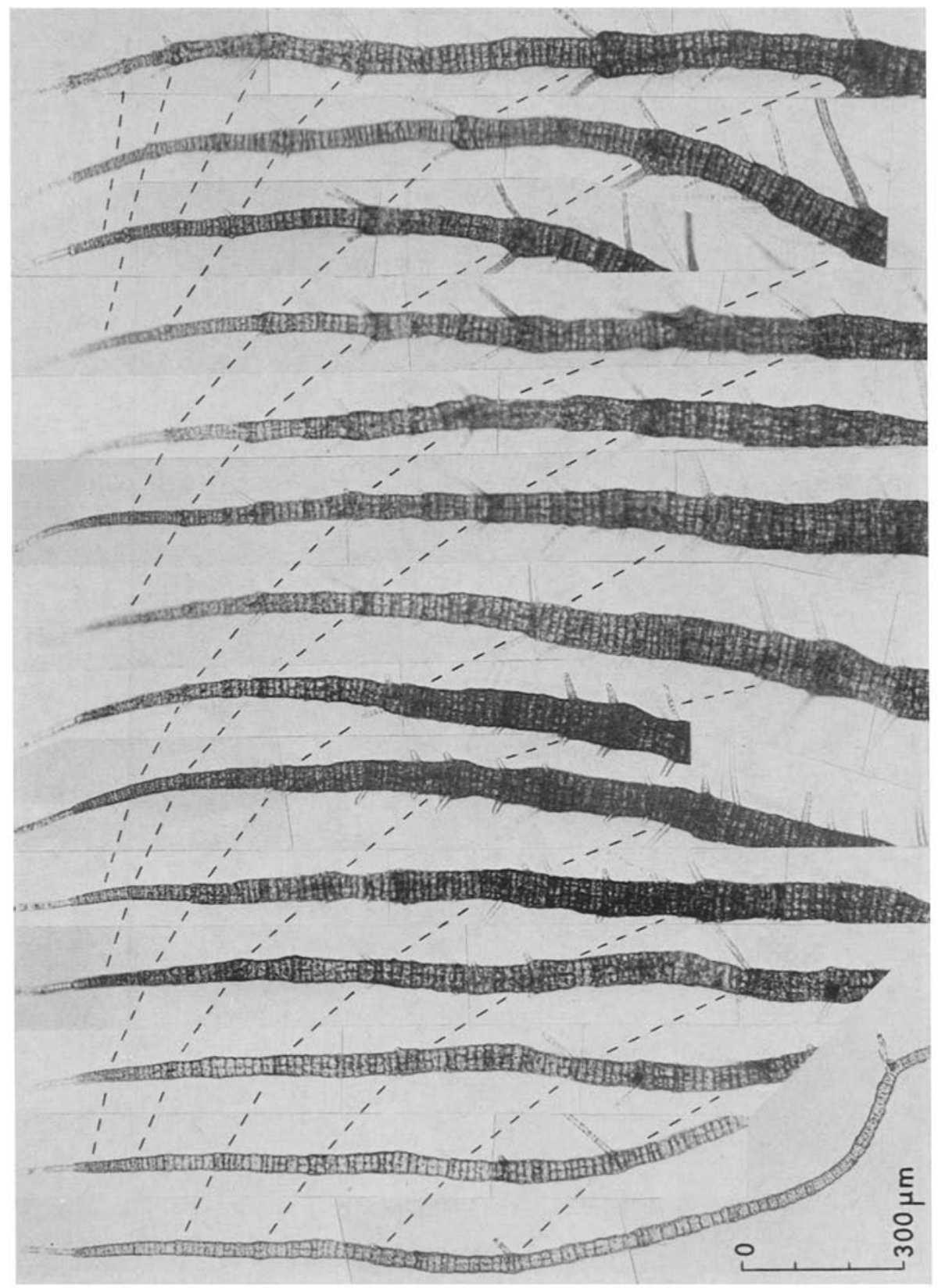

Abb. 8: Striaria attenuata. Wachstum einer Sproßspitze bis zur Längsteilung ihrer Endzelle. Aufnahmen in Tagesabständen 


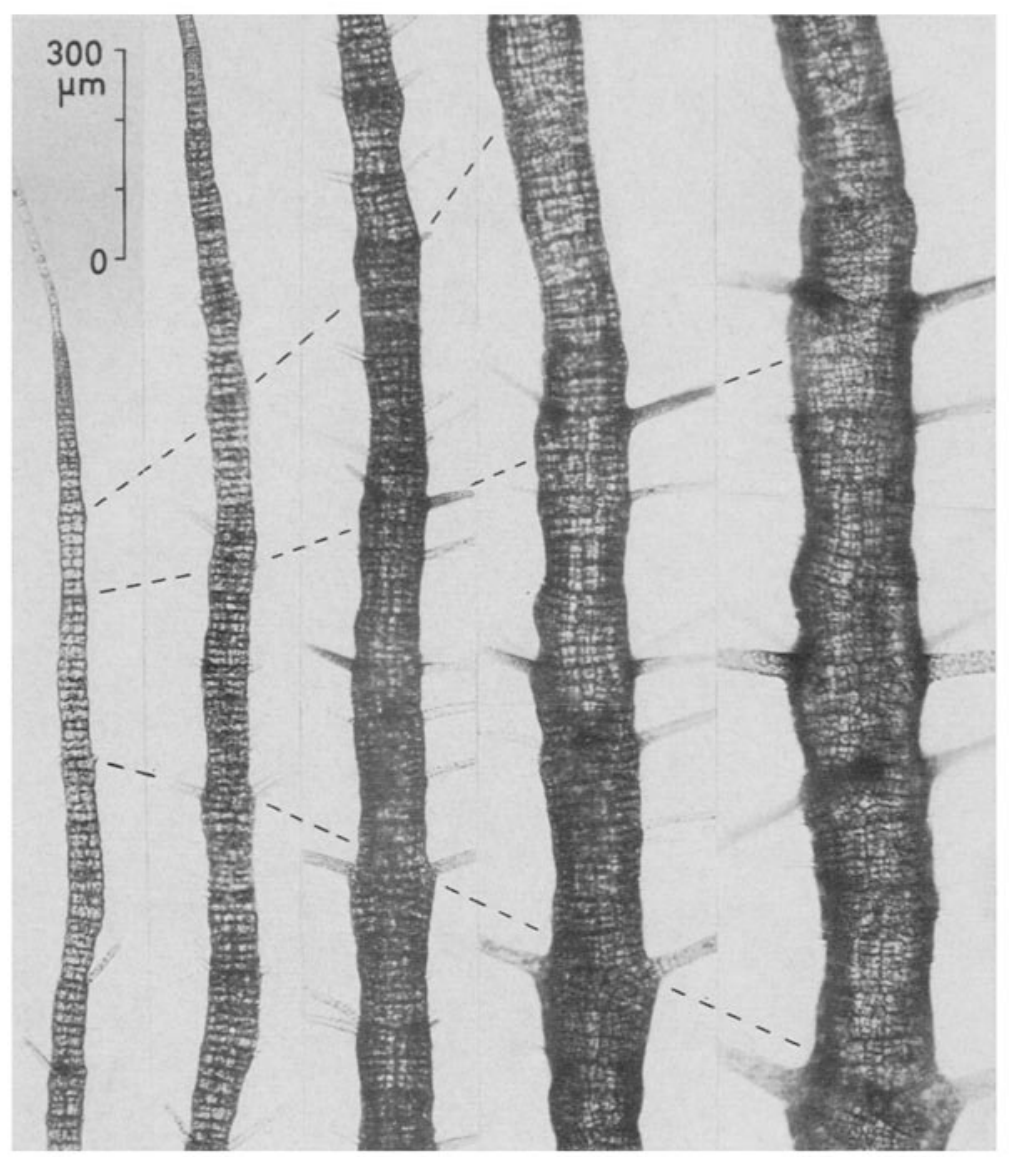

Abb. 9: Striaria attenuata. Dickenzunahme eines Achsenabschnittes, jeweils in Abständen von zwei Tagen aufgenommen

zum Schluß der Serie, in der die Längsteilungen auch dic oberste Zelle des monosiphonen Fadenendes erreichen, streckt sich der Spitzenabschnitt mit gleicher Geschwindigkeit.

Die Wachstumsanalyse zeigt also mit aller Deutlichkeit, daß ein interkalares Meristem bei Striaria nicht vorhanden ist, wie es nach Chadefaud (1960, Fig. 194,5) den Punctariaceen, Scytosiphonaceen und verwandten Familien eigen sein soll. Auch HameI (1931-1939) gibt für Striaria eine interkalare Wachstumszone unterhalb der Thallusenden an, und ebenso schreibt GaYral (1966) der Alge ein trichothallisches Wachstum zu.

Ganz stetig wird auch der hohl gewordene Striaria-Thallus dicker. In Abbildung 9 - einem mittleren Abschnitt aus der bereits in der vorhergehenden Serie dargestellten Pflanze - folgen die Aufnahmen einander in Abständen von zwei Tagen. Die Rindenzellen teilen sich in beiden Richtungen, vorzugsweise jedoch quer. Das Verhältnis von Streckung und Dickenzunahme ist längs der Achse nicht ganz einheitlich. 
Aus den Rindenzellen gehen die Initialen für Zweige und Haare hervor. Die kräftigsten Seitenzweige sind die primär an der noch nicht oder gerade hohl gewordenen Achse angelegten, während die später aus der schon weiter differenzierten Rinde hervorgegangenen $Z$ weige schwächer bleiben. Häufig entstehen die $Z$ weige, stets aber die Haare wirtelig an dem oberen Ende der tonnenförmigen Thallusabschnitte. Ganz offensichtlich ist der charakteristische stockwerkartige Aufbau der Achsen in der ringförmigen Anlage von Zweig"- und Haarinitialen begründet. Thre Ursprungszellen teilen sich nämlich nicht mehr wie die übrigen Rindenzellen, daher bleibt der Thallusumfang in den Zonen der haartragenden Wirtel kleiner. Nicht immer tritt diese Struktur so $\mathrm{klar}$ in Erscheinung wie in Abbildung $3 \mathrm{C}$, die einen Zweigabschnitt aus einer völlig ruhigstehenden Kultur zeigt. Die Haarwirtel werden leicht abgeworfen; in diesen Zonen entstehen später die Sporangien (Abb. $3 \mathrm{~B}$ ), deren gürtelförmig streifiger Anordnung die Gattung ihren Namen verdankt. Und schließlich kann man sogar noch in den tonnenförmigen Stockwerken des fertigen Thallus die Segmente seines ursprünglich monosiphonen Stadiums wiedererkennen (Abb. 7). So enthïllt uns die Beobachtung seiner Entstehungsgeschichte die klare Gesetzmäßigkeit des nur scheinbar so unregelmäßig aufgebauten Striaria-Thallus.

Ob sich wohl auch die Thalli von Ilea und Scytosiphon in entsprechender Weise entwickeln? Wir haben es nicht geprüft, aber es ist doch wahrscheinlich.

\section{ZUSAMMENFASSUNG}

1. Striaria attenuata wurde 1971 erstmalig bei Helgoland beobachtet.

2. In Kultur folgten mehrere Generationen mit unilokulären Sporangien aufeinander.

3. Die Zoosporen entwickeln sich zu kriechenden, später halbkugeligen Protonemata. Nur auf einem geringen Teil - etwa einem von 70 - entstehen im Alter von 3 Wochen aufrechte Sprosse.

4. Das Protonema hat Spitzenwachstum, die Striaria-Thalli wachsen auf ihrer ganzen Länge interkalar ohne Ausbildung einer meristematischen Zone.

\section{ZITIERTE LITERATUR}

Caram, B., 1965. Recherches sur la reproduction et le cycle sexué de quelques Phéophycées. Vie. Milieu 16 (A), 21-226.

Chadefaud, M., 1960. Traité de Botanique Systérnatique. Masson, Paris 1, 1-1018.

Gayrai, P., 1966. Les algues des côtes françaises (Manche et Atlantique). Doin-Deren, Paris, $632 \mathrm{pp}$.

Hamel, G., 1931-1939. Phéophycées de France. Paris, XLVII + 432 pp.

KylrN, H., 1934. Zur Kenntnis der Entwicklungsgeschichte einiger Phaeophyceen. Lunds Univ. Årsskr. N.F. (Ard. 2) 30 (9), 1-19.

RernKe, J., 1878. Uber die Entwicklung von Phyllitis, Scytosipbon und Asperococcus. Jb. wiss. Bot. 11, 262-273.

Anschrift des erstgenannten Autors: Dr. Dr. h. c. P. Kornmann Biologische Anstalt Helgoland (Meeresstation)

2192 Helgoland

Bundesrepublik Deutschland 